\title{
WBC Aplasia: Case Report
}

\begin{abstract}
Pure white cell aplasia (PWCA) is a rare acquired cause of agranulocytosis characterized by absence of all myeloid lineages in bone marrow with intact erythropoiesis and megakaryopoiesis. Reported cases were associated with autoimmune conditions, thymomas, chronic lymphocytic leukemia, infections, and as an adverse drug reaction. Presentation ranges from asymptomatic severe neutropenia to fatal sepsis. Several medications were tried along with treatment of the underlying cause if identified. Steroids, immunoglobulin, azathioprine, cyclosporine, rutiximab, GCSF and bone marrow transplant were all tried with variable response. Here we report a case of PWCA in middle age male, who responded to cyclosporine.
\end{abstract}

Volume 8 Issue 6 - 2020

\author{
Mariam AlGhazal,' Rehab Bu Khamseen,' \\ Mona AlZaher, ${ }^{2}$ Lama AlGharyafi, ${ }^{2}$ Zainab \\ Alruwaiy, ${ }^{2}$ Manal alghazal' \\ 'Department of hematopathology Ministry of health, Dammam \\ regional laboratory \& blood banking, Saudi Arabia \\ 2Department of hematology Dammam medical complex \\ hospital, Saudi Arabia
}

\section{Correspondence: Mariam AIGhazal, Department of hematopathology Ministry of health, Dammam regional laboratory \& blood banking, Saudi Arabia, \\ Email dr.m.r.alghazal@hotmail.com}

Received: November 12, 2020 | Published: November 26, 2020

\section{Introduction}

Pure white cell aplasia (PWCA) is a rare cause of neutropenia and mechanisms remain uncertain. The diagnosis is based on bone marrow examination, which reveals virtually absent granulopoiesis, while erythropoiesis and megakaryocytopoiesis are intact. ${ }^{1}$ Clinically, patients with PWCA present with severe neutropenia and recurrent infections. The peripheral smear and cell count show only severe neutropenia. PWCA may be drug-induced and cases have also been reported in association with autoimmune diseases and thymomas. Immunologic mechanisms have been demonstrated in some PWCA cases where an inhibiting antibody was found Treatment with thymectomy or drug discontinuation may be clinically useful Other cases have required immunosuppressive therapies including steroids intravenous immunoglobulin (IVIG), cyclosporine (CsA) or rituximab with A Bone marrow transplantation has also been utilized with some success. ${ }^{2}$

\section{Case presentation}

We have admitted to our hospital a 46 years old Male, from Sudan, came complaining of subjective fever for 3 days duration. His condition was associated with sore throat, arthralgia and myalgia. No history of headache, skin rashes, no weight loss or decrease in appetite, gastrointestinal symptoms or respiratory symptoms. His past medical history was negative except for one episode of malaria years back $\&$ he was treated there empirically. Family history was negative for similar condition or any history of malignancy or autoimmune disease. Examination revealed thin built, well looking middle age male not pale or jaundiced no lymph node palpable, no skin rashes throat was mildly congested. Other systemic examination was unremarkable Peripheral blood examination show that WBC: $2.440 \times 10^{9} / \mathrm{L}$ (normal range $\left.4.0-11.0 \times 10^{9} / 1\right)-\mathrm{HB}: 14.4 \mathrm{gm} / \mathrm{dl}$ platelets: $354,000 / \mathrm{ul}($ normal range is $150-450,000 / \mathrm{ul})$. There is marked neutropenia with absolute neutrophil count of $0 / \mathrm{ul}$ (virtual absence of neutrophils). Total lymphocytes are around $90 \%$ no evidence for blasts or abnormal population except for reactive lymphocytes \& some LGL lymphocytes (19\%). Bone marrow studies showParticulate hypocelluar bone marrow aspirate with markedly diminished myeloid precursors, (there are only $2-3 \%$ promyelocytes seen with virtual absence of any late myeloid precursors and neutrophils. Erythropoiesis constitutes $45 \%$ of the total hematopoiesis and basically normoblastic in maturation. Megakaryocytes are present \& normal in morphology Mature lymphocytes and plasma cells constitutes $50 \%$ and $2 \%$ respectively (there is around $11 \%$ large granular lymphocytes with abundant cytoplasm \& fine azurophilic granules out of total lymphocytes). There is around $2 \%$ blast like or hematogones in addition to few scattered histiocytes with hemophagocytic activity \& rare mast cell.

No evidence of abnormal infiltration or hemoparasites seen in the material studied. Flowcytometry conducted on bone marrow aspirate shows almost absent scatter in the granuloctic region (virtual absence of CD10 in the granulocytes gate denoting absent mature myeloid). Analysis of cells in the lymphocytes gate shows Lymphocytes to be around $54 \%$. CD3 positive T-lymphocytes constitute $84 \%$. CD8 is higher than CD4 (77.5\% vs $20.8 \%$ alternatively) with normal expression of CD2, CD5 but diminished expression of CD7. However CD8 positive lymphocytes show Absent CD7 in 30\% of cells and CD57 positivity in $50 \%$ of cells. There is around $12 \%$ NK lymphocytes Hematogones constitute around $3 \%$ and majority are stage II which is positive for both CD19, CD10. Other investigations including renal and Liver functions are normal Abdomen ultrasound -normal study So Patient was started on fourth generation cephalosporins. All Investigations that ordered for HIV, hepatitis, malaria screen, CMV, EBV, brucella, salmonella were negative. All septic work up is negative. Autoimmune profile shows ANA 1:80 Rheumatoid factor negative.

The Patient remained afebrile but persistently neutropenic for more than 2 weeks, so bone Since most of the secondary causes were ruled out, Chest CT with contrast to role out mediastinal mass such as thymoma was done and the study was normal. So at this point we were left with either autoimmune cause of PWCA or idiopathic. So antineutrophil antibodies requested and came negative Immunoglobulins level. All diagnostic work up for secondary underlying causes were found to be negative and he is diagnosed as idiopathic PWCA. Patient received GCSF injection in combination with cyclosporine (cyclosporine $3 \mathrm{mg} / \mathrm{kg} /$ day in bid doses), after starting treatment by 2 days there was marked improvement in WBC jump to 3.79 and the next day was 4.91 as well as neutrophil count improve and reach up to 1.84 , therapy was successful to induce neutrophil recovery. He was discharged on cyclosporine and seen after 4 days in clinic and the 
dose was increased to maintain level 200-400. Initially the neutrophils count drop to which indicate that the initial response was secondary to GCSF. He then showed excellent response to cyclosprine single therapy with neutrophils.

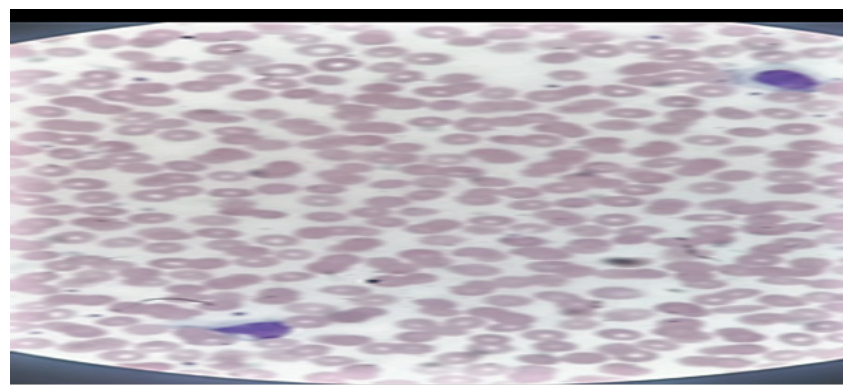

Figure I Peripheral blood morphology shows virtual absence of neutrophils \& 2 large granular lymphocytes.

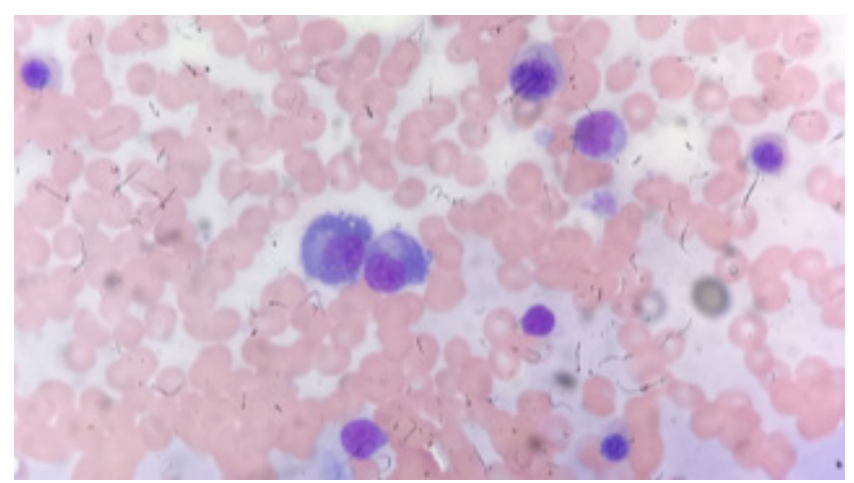

Figure 2 Bone marrow aspirate shows hardly myeloid cells, plasma \& erythroid cells are seen.

\section{Discussion}

This case is thought to be an idiopathic wbc aplasia, as all other possibilities are excluded. In fact this is the first case seen in our hospital, since the patient is from sudan, then it might be not be common in our population or may be underdiagnosed.

After investigations he was considered as idiopathic WBC aplasia. The patient was treated with combined GCSF and cyclosporine, then maintained on cyclosporine alone. For now, the suggested proposal for identifying pure white cell aplasia is the finding of isolated profound neutropenia in the context of a marrow examination showing virtual absence of granulopoiesis. A reasonable work-up should exclude thymomas, autoimmune disease, infectious etiologies, and drug effects ${ }^{2}$ even the terminology is relatively not common as RBC aplasia. The exact pathological mechanism of pure white cell aplasia is unclear. Evidence from a limited number of cases has suggested that specific auto-antibody inhibition of receptors involved in myelocyte maturation play a key role.

\section{Conclusion}

WBC aplasia is one of the rare aplasia \&still the mechanism is uncertain, facing such situation, bone marrow examination is essential to confirm such diagnosis, reporting such cases is important \& exclude all the possible etiologies.

\section{Conflicts of interest}

The authors declare that they have no conflicts of interest.

\section{Author contribution}

Dr. Mariam Al Ghazal, was involved in diagnosis while Dr. Mona AlZaher was involved in the investigations, treatment \& follow up. Dr. Alghazal defined the manuscript while Dr. Mona made the reviews with valuable input. All Authors read and approved the final manuscript.

\section{References}

1. Sadia Sultan, Syed Mohammad Irfan. Acquired idiopathic pure white cell aplasia: A rare cause of agranulocytosis. Journal of applied hematology. 2014;5(4):161-166.

2. David M Nguyen, Rondeep Brar Stanley L Schrier. The Varying Clinical Picture of Pure-White Cell Aplasia. Journal of Blood Disorders \& Transfusion. 2014;5:218.

3. Thomas Keast, Deshan Weeraman, Phil Mayhead, et al. Pure white cell aplasia: report of first case associated with autoimmune hepatitis. Frontline Gastroenterol. 2014;5(4):287-290. 